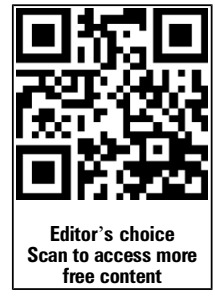

free content

\title{
Fifteen-minute consultation on limiting investigations in the fainting child
}

\section{Michael Harris, Frances Bu'Lock}

Department of Paediatric Cardiology, University Hospitals Leicester NHS Trust, Leicester, UK

\section{Correspondence to} Dr Michael Harris, Paediatric Cardiology, University Hospitals Leicester NHS Trust, Glenfield Hospital, Groby Road, Leicester LE3 9QP, UK; michaeljharris@doctors.org.uk

Received 6 April 2015 Revised 24 June 2015 Accepted 2 July 2015 Published Online First 22 July 2015
CrossMark

To cite: Harris M, Bu'Lock F. Arch Dis Child Educ Pract Ed 2016;101:26-30.

\begin{abstract}
Syncope can be a dramatic and frightening experience for the patient and onlookers. It can also be a confusing area for doctors, with a lack of diagnostic certainty and a differential that ranges from the completely benign to the lifethreatening. This is a tricky area for clinicians, who can find it difficult to reassure their patients and their families. The aim of this article is to present a clear scheme for appropriate investigation and referral of paediatric patients with syncope. The aim of investigations in such cases should be to confirm or exclude serious, life-threatening causes of syncope.
\end{abstract}

Syncope can be a dramatic and frightening experience for the patient and onlookers. It can also be a confusing area for doctors, with a lack of diagnostic certainty and a differential that ranges from the completely benign to the lifethreatening. Referrals for tertiary opinions occupy a lot of time in paediatric cardiology and neurology clinics $(5.8 \%$ of paediatric cardiology clinic referrals in a 1 year period in a US Centre). ${ }^{1}$ Recent well publicised events on the sports field have added to the anxiety. The primary concern is generally whether or not the syncopal episode is an event that precedes sudden cardiac death or signals the onset of epilepsy. Although sudden cardiac death is rare (0.6-8.5 deaths/ 100000 patient years $)^{1}$ it occupies a disproportionate amount of the public and professional mind.

This is a tricky area for clinicians, who can find it difficult to reassure their patients and their families. The aim of this article is to present a clear scheme for appropriate investigation and referral of paediatric patients with syncope. The aim of investigations in such cases should be to confirm or exclude serious, lifethreatening causes of syncope. ${ }^{2}$

\section{WHAT IS SYNCOPE?}

Syncope is a transient, but complete, loss of consciousness (with associated loss of muscle tone) due to global cerebral hypoperfusion. ${ }^{3}$ By definition:

- It is of rapid onset

- It is of short duration

- Recovery is

- quick

- spontaneous

- complete

Therefore, by definition, this excludes:

- Falls, which usually do not involve loss of consciousness

- States of altered, but not loss of, consciousness

- Coma, which, by definition, is not transient loss of consciousness

- Aborted sudden cardiac death

- Epileptic seizures

- Psychogenic causes

- 'Near' syncope, in which patients do not experience loss of consciousness, but can have several symptoms including lightheadedness, etc.

\section{WHAT CAUSES SYNCOPE?}

Syncope can be divided on an aetiological basis, into

- Syncope due to cardiac causes

- Syncope due to reflex mechanisms, which includes vasovagal syncope

- Orthostatic hypotension.

\section{WHY DOES SYNCOPE HAPPEN?}

Syncope occurs when the systolic blood pressure (BP) falls below around $60-70 \mathrm{~mm} \mathrm{Hg},{ }^{2}$ although this exact figure is age-related.

BP is the product of cardiac output and systemic vascular resistance. Therefore, anything that affects one or both of these determinants will cause an alteration in BP. Examining cardiac output and systemic vascular resistance in turn. 
Cardiac output can be affected by:

- Cardiac mechanisms

- Changes in heart rate and rhythm

- Structural heart problems, for example, aortic stenosis

- Reflex (nervous) mechanisms

- Slow heart rate due to reflex nervous activity (vagal)

- Orthostatic mechanisms

- Inadequate venous return

Systemic vascular resistance can be affected by:

- Reflex mechanisms

- Vasodilation due to inappropriate reflex activity

- Orthostatic mechanisms

- Impairment, due to a variety of primary and secondary causes, of the autonomic nervous system

- Generally cardiac factors do not affect systemic vascular resistance (figure 1).

\section{WHAT CAUSES CARDIAC SYNCOPE?}

The serious, life-threatening causes of syncope are generally cardiac in nature. Cardiac causes of syncope result in low cardiac output. Cardiac syncope may be due to:

- Electrical disturbances (arrhythmias)

- Bradycardia, which is uncommon in children

$\bigcirc$ Sinus node dysfunction

$\bigcirc$ AV node dysfunction

Implanted device (eg, pacemaker) malfunction

- Tachycardia

○ Supraventricular

○ Ventricular

Long QT syndrome ${ }^{4}$ (see figure 2)

Brugada syndrome (see figure 3 )

Catecholaminergic polymorphic ventricular tachycardia (see figure 4)

- Drug-induced, which may act via the above mechanisms.

- Structural cardiac problems

- Hypertrophic cardiomyopathy

- Dilated cardiomyopathy

- Arrhythmogenic right ventricular cardiomyopathy

- Coronary artery anomalies

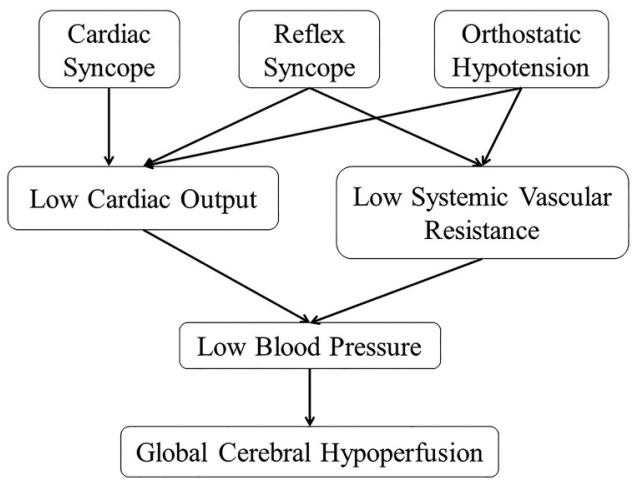

Figure 1 Mechanisms of syncope.

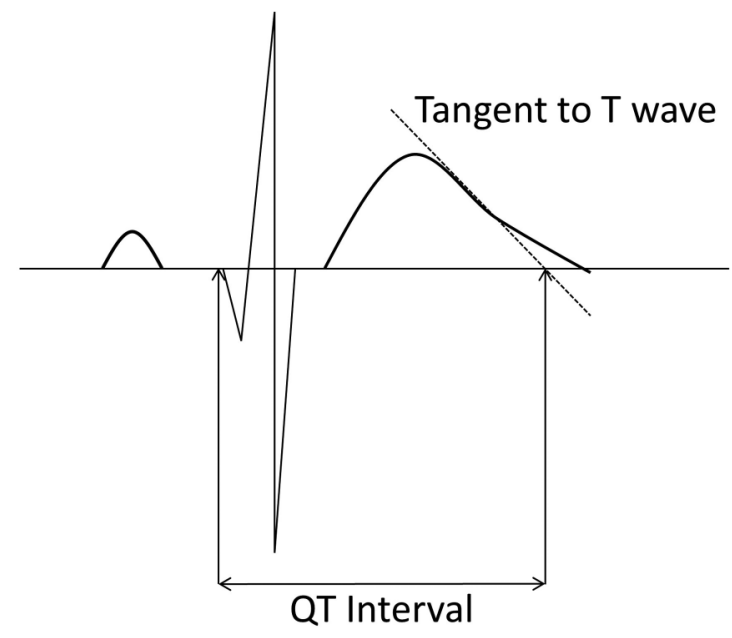

Bazett's Formula: QTc $=$ QT $/$ VPreceding RR

Figure 2 ECG of long QT syndrome showing the 'Tangent' method for measuring QT interval.

○ For example, left coronary artery coursing between the aorta and the pulmonary artery; anomalous origin of the left coronary artery from the pulmonary artery

- Aortic stenosis, resulting in an obstructed left ventricular outflow tract

- Myocarditis

- Pulmonary hypertension, although there is usually a history of exertional dyspnoea rather than syncope in this condition

- Cardiac tumours

The non-life-threatening causes of syncope, therefore, fall into the remaining two categories, namely:

- Reflex syncope, which includes 'vasovagal' episodes

- Orthostatic hypotension.

\section{HOW SHOULD YOUNG PEOPLE WITH SYNCOPE BE ASSESSED?}

Assessment begins with an accurate history to confirm syncope, to identify a possible causal mechanism (cardiac, reflex, orthostatic-see box 1 for further characteristics) and to ascertain any relevant background information, including family history. Assessment should

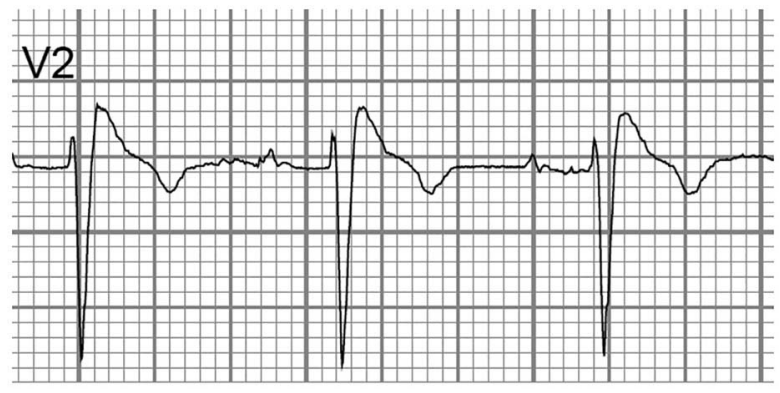

Figure 3 ECG showing characteristic appearance of ST segment in Brugada syndrome. 


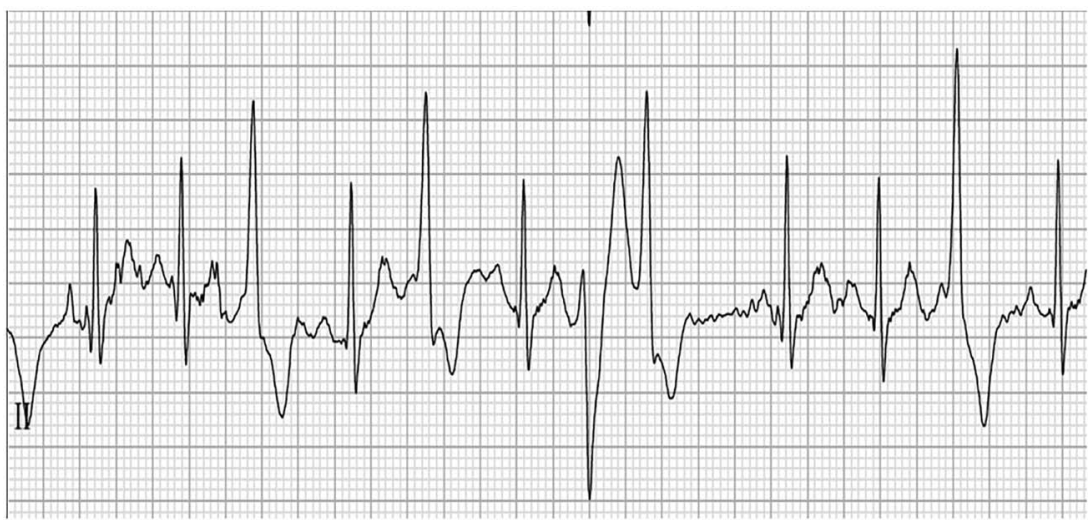

Figure 4 ECG showing appearances of polymorphic ventricular complexes on exercise testing in a patient who is $\beta$-blocked and known to have catecholaminergic polymorphic ventricular tachycardia.

continue with physical examination. For completeness, BP can be recorded at this time. A 12-lead ECG should be performed as the initial baseline investigation in syncope. On the basis of these initial actions, further investigations or onward referral can be considered.

Studies have confirmed that the sensitivity of screening using history, examination and 12-lead ECG findings can be $100 \%$ sensitive for the cardiac causes of syncope. ${ }^{15}$

\section{HISTORY}

The mainstay of diagnosis is in the history. First and foremost, loss of consciousness must be confirmed. Many patients have funny turns but not true syncope. The event should be transient, of rapid onset and short duration. There should be loss of postural tone. Recovery should be spontaneous, complete and without sequelae. Lack of an eyewitness can make diagnosis more challenging. If not all of these features can be confirmed, then it will be necessary to exclude other causes of loss of consciousness. Seizures may be a sequelae of syncope. However, it is important to note that a number of people diagnosed with epilepsy may in fact have a cardiac cause of syncope. In a study from New Zealand, several patients had a delayed diagnosis

Box 1 Classical premonitory symptoms for syncope $^{2}$

Often evolving, gradual, progressive

- Nausea

- Blurred/tunnel vision

- Hearing changes (muffled, buzzing)

Dizziness

Light-headedness

Sweating

Pallor

Cold, clammy skin

Weakness, tremulousness of long QT syndrome and were instead diagnosed as epileptic. On review, several of these patients had had baseline ECGs that were abnormal, but because the corrected QT had been miscalculated initially, the diagnosis had been missed. ${ }^{6}$ The corrected QT interval should be calculated using Bazett's formula, which corrects for heart rate. The end of the Twave can be difficult to define, but using the 'Tangent' method, this uncertainty is much reduced ${ }^{4}$ (see figure 2 ).

Once syncope is confirmed, the history must seek to elucidate the possible cause, that is, cardiac, reflex or orthostatic. A good history of the event, the situation and position of the patient before, during and after the event should be sought (see boxes 1-4). If mobile phone or video footage of the event exists, then this is often invaluable.

In the background, a family history of sudden death, arrhythmias at a young age (less than 40 years) and previous cardiac disease should be confirmed or excluded. Of note, a study from the Netherlands published in 2009 showed that patients with long QT syndrome were significantly more likely to have a family history of syncope and sudden death. ${ }^{7}$ Neurological disorders, including epilepsy and other neurodisabilities, and metabolic problems, principally diabetes, should be sought. Medications, including vasoactive substances such as diuretics, and other drugs (including of abuse) should be assessed.

Box 2 Features suggestive of reflex (neurally mediated) syncope 238

Nausea (unique to non-arrhythmogenic syncope)

- Vomiting

- After an emotionally stressful event

- Following prolonged standing in a hot, crowded area

- During or after a meal

- After (not usually during) exercise

- With head rotation 
Box 3 Features suggestive of syncope due to orthostatic hypotension ${ }^{3}$

Following standing from supine, sitting or squatting position

Standing after exercise

Prolonged standing in a hot environment

Secondary to commencement of vasoactive medications

\section{RED FLAGS FOR CARDIAC SYNCOPE}

More specifically, 'Red Flag' features in the history significant for a cardiac cause of syncope are shown in box 4 . Some investigators ${ }^{8}$ have found that dizziness is a symptom in patients with arrhythmogenic (cardiac) syncope. However, nausea appears to be unique to non-arrhythmogenic syncope.

Reflex syncope is suggested by typical triggers and typical prodromal symptoms (see boxes 1 and 2). The European Society of Cardiology ${ }^{3}$ further subdivides reflex syncope into vasovagal, situational, carotid sinus and atypical syncope.

Vasovagal syncope occurs secondary to emotional stressors or the sight of blood, or a painful stimulus, among others, but can also occur secondary to orthostatic stress. For example, this would include syncope on standing from a lying or squatting position.

Situational syncope might occur secondary to ordinary activities such as stretching, hair brushing, coughing, sneezing, micturition, swallowing (especially cold liquids), defaecation, following meals, after prolonged standing, in warm or crowded environments, ${ }^{9}$ or immediately after (not during) strenuous exercise.

Carotid sinus syncope is presumably secondary to carotid sinus massage causing vagal stimulation. This might occur when wearing tight collars or neckties.

Atypical forms of reflex syncope also occur. These are, by definition, rather difficult to define, but occur without apparent triggers or with an atypical presentation.

\section{Box 4 Red flag symptoms for cardiac syncope 37}

During exertion

- When supine

When asleep

- Immediately following sudden onset palpitations

- Exertional chest pain prior to syncope

In a swimming pool

Secondary to auditory stimuli

Without warning (no prodrome)

- Structural heart disease

- Family history of:

- Sudden death

- Congenital arrhythmias
Orthostatic hypotension (see box 3) can be thought of as a spectrum of several conditions, some of which are more common in adults and the elderly. ${ }^{3}$ While the pathophysiological mechanisms are different, there are similarities in clinical symptomatology between reflex syncope and orthostatic hypotension. In some situations, orthostatic hypotension can trigger reflex vasovagal mechanisms, causing syncope. This occurs more commonly in younger patients than in the older population. Initial hypotension, causing symptoms of a 'head rush', is common in adolescents upon standing, although syncope is rare. If syncope due to orthostatic hypotension occurs in the paediatric population, it is more likely due to volume depletion in the context of haemorrhage, diarrhoea or vomiting. Drugs, including vasodilators and diuretics, may induce hypotension. Alcohol should not be forgotten, although intoxication is a more likely cause for apparent syncope. Apart from this, primary and secondary autonomic failure is more common in adults.

\section{POSTURAL ORTHOSTATIC TACHYCARDIA SYNDROME}

Syncope is not a part of this increasingly recognised syndrome. ${ }^{10}$ Postural orthostatic tachycardia syndrome is characterised by orthostatic symptoms (ie, light-headedness, dizziness, vision changes, nausea), which occur on a daily basis and, for diagnostic purposes, should have persisted for several months, coupled with sympathetic activation (excessive heart rate, palpitations, chest pain). In contrast to true orthostatic intolerance, orthostatic hypotension is absent. ${ }^{11}$ In this context, excessive heart rate in children and teens is defined as an increase above baseline of greater than $40 \mathrm{bpm}$. Some investigators use a head-up tilt test as part of their diagnostic protocol. The ECG should show sinus rhythm. Symptoms should improve with recumbence. Female patients outweigh men by $3-4: 1$, with a high preponderance of chronic fatigue and gastrointestinal dysfunction as comorbidities. A variety of treatments, including medications, are available to treat patients with postural orthostatic tachycardia syndrome, but one of the keys is exercise reconditioning.

\section{LIMITING INVESTIGATIONS IN YOUNG PEOPLE WITH SYNCOPE}

Cardiac causes of syncope can most likely be excluded with high sensitivity with appropriate history taking, clinical examination and a 12-lead ECG. Further investigations are probably inappropriate. It may be helpful, and indicated, to obtain an ambulatory ECG prior to referral to, or at the suggestion of, a paediatric cardiologist. However, ambulatory ECG monitoring has a low yield ${ }^{3}$ for capturing arrhythmia, of the order of $14 \%{ }^{1}$ in some studies, even in those subsequently shown to have cardiac syncope. In the same 
study, $15 \%$ of patients reported symptoms that had no correlation on ambulatory ECG monitoring. The low yield is likely due to the relative infrequency of arrhythmia events, which are too infrequent to occur contemporaneously with monitoring.

Further investigations that may be carried out by a cardiologist may include an echocardiogram, in the context of an abnormal clinical cardiovascular examination, exertional syncope or family history of heart muscle disease. In cases of exertional syncope, an echocardiogram is probably warranted to exclude such rare anatomical anomalies as anomalous origin of the left coronary artery from the pulmonary artery. The diagnostic yield of echocardiography is low, as with ambulatory ECG monitoring, less than $50 \%$ in some studies, even in those with cardiac syncope. In one study from several years ago, ${ }^{12}$ the sensitivity of echocardiography for detecting a cardiac cause of syncope was $18 \%$.

In the case of exertional symptoms or startle-related syncope, exercise testing is mandated, as conditions such as catecholaminergic polymorphic ventricular tachycardia cannot be detected on a resting ECG. ${ }^{13}$

Other, further investigations might include:

- Longer-term ambulatory monitoring, dependent upon symptom type and frequency. Event monitors (patient activated) generally have no role in syncope, but are useful in palpitations

- Implantable loop recorder

- Invasive electrophysiological studies, although these are rarely required

- Tilt-table testing is not particularly useful in the paediatric population, and should be left in the hands of experts. In the context of limiting investigations in fainting children, tilt testing is not usually required for excluding the life-threatening causes of syncope. It may be helpful, in the hands of experienced investigators, in discriminating between reflex syncope, orthostatic hypotension, seizure disorders and psychiatric disorders. Protocols for tilt testing are not uniform, and as children have a higher propensity to orthostatic hypotension compared with the adults for whom the protocols were designed, there is a lack of specificity inherent in this investigation.

In summary, when looking to limit investigations in the fainting child, it is important to have as clear as possible a history of the events. If syncope is confirmed, then the object is to exclude serious, potentially life-threatening, causes of syncope. These are essentially the cardiac causes of syncope. ${ }^{13}$

History, examination and ECG are usually sufficient to confirm benign causes of syncope, or to identify those patients who need referral for further assessment and investigation.

Fainting in children will continue to provoke consternation, and there will always be diagnostic dilemmas. However, the vast majority of syncopal episodes in children are benign in nature, and it is satisfying and resource efficient to be able to swiftly reassure children and their parents on the basis of history, clinical examination and some rudimentary investigations.

Contributors $\mathrm{MH}$ researched and wrote the article. $\mathrm{FB}$ reviewed and edited the article.

Competing interests None declared.

Provenance and peer review Commissioned; externally peer reviewed.

\section{REFERENCES}

1 Tretter JT, Kavey REW. Distinguishing cardiac syncope from vasovagal syncope in a referral population. J Pediatr 2013;163:1618-23.

2 Moodley M. Clinical Approach to syncope in children. Semin Pediatr Neurol 2013;20:12-17.

3 Moya A, Sutton R, Ammirati F, et al. Guidelines for the diagnosis and management of syncope (version 2009). Eur Heart J 2009;30:2631-71.

4 Postema PG, De Jong JS, Van der Bilt IA, et al. Accurate electrocardiographic assessment of the QT interval: teach the tangent. Heart Rhythm 2008;5:1015-18.

5 Johnson ER, Etheridge SP, Minich LL, et al. Practice variation and resource use in the evaluation of pediatric vasovagal syncope: are pediatric cardiologists over-testing? Pediatr Cardiol 2014;35:753-8.

6 MacCormick JM, McAlister H, Crawford J, et al. Misdiagnosis of long QT syndrome as epilepsy at first presentation. Ann Emerg Med 2009;54:26-32.

7 Colman N, Bakker A, Linzer M, et al. Value of history-taking in syncope patients: in whom to suspect long QT syndrome? Europace 2009;11:937-43.

8 MacCormick JM, Crawford JR, Chung SK, et al. Symptoms and signs associated with syncope in young people with primary cardiac arrhythmias. Heart Lung Circ 2011;20:593-8.

9 Wieling W, Ganzeboom KS, Saul JP. Reflex syncope in children and adolescents. Heart 2004;90:1094-100.

10 Stewart JM. Common Syndromes of Orthostatic Intolerance. Pediatrics 2013;5:968-80.

11 Jarjour IT. Postural tachycardia syndrome in children and adolescents. Semin Pediatr Neurol 2013;20:18-26.

12 Ritter S, Tani LY, Etheridge SP, et al. What is the yield of screening echocardiography in pediatric syncope. Pediatrics 2000;105:1-3.

13 Bat T, Collins KK, Schaffer MS. Syncope during exercise: just another benign vasovagal event? Curr Opin Pediatr 2011;23:573-5. 